\title{
Editorial
}

\section{Of Models and Methods: Our Analytic Armamentarium Applied to Methicillin-Resistant Staphylococcus aureus}

\author{
Eli N. Perencevich, MD, MS; David M. Hartley, PhD
}

To study, and when the occasion arises to put what one has learned into practice-is that not deeply satisfying?-Confucius, The Analects

The purpose of computing is insight, not numbers.-R. W. Hamming

Pandemic Staphylococcus aureus and methicillin-resistant Staphylococcus aureus (MRSA) emerged as serious nosocomial pathogens years ago. ${ }^{1}$ Despite considerable attention in the literature and in clinical practice, the problem continues unabated. MRSA-associated morbidity and mortality is, in fact, increasing, as are the associated healthcare costs. This issue of Infection Control and Hospital Epidemiology contains four studies that explore nosocomial $S$. aureus and MRSA, approaching the problem using a variety of methodologies including a quasi-experimental design, a case-control study, a cohort study, and mathematical models. $^{2-5}$ The diversity of approaches illustrates the impressive armamentarium at our disposal to meet the challenge of understanding the epidemiology of nosocomial infections. Furthermore, it highlights the challenges to hospital epidemiologists, who constantly face new data to interpret and incorporate into practice.

The study by Khoury et al., a combined quasi-experimental and case-control study, describes an outbreak of MRSA infections in a neonatal intensive care unit (NICU) and subsequent successful eradication of a clonal strain after aggressive surveillance culturing and control interventions. ${ }^{4}$ The multi-modal interventions included admission and weekly active surveillance culturing of the periumbilical and perirectal skin of all infants with subsequent contact isolation (gowns, gloves, and, occasionally, masks) of MRSA carriers; swabs of the anterior nares of all NICU employees with aggressive decolonization and repeat culture if they were found to be colonized; and direct observation and education of healthcare workers (HCWs) in proper contact isolation and hand hygiene practices. Using a case-control study, Khoury et al. identified several univariate unadjusted risk factors in their comparison of 12 patients identified as having MRSA infections with random control-patients. Multiple gestation, gavage feeding, intubation, younger gestational age at birth, and lower birth weight were all found to be associated with MRSA infection. In addition, when they compared the patients found to be colonized on active surveillance swabs with the same control-patients, they found multiple gestation, younger gestational age at birth, and lower mean birth weight to be associated with MRSA colonization. The authors also reported a significantly longer NICU stay for MRSA-infected patients compared with control-patients; however, it was unclear whether this length of stay represented a risk factor for, or an outcome of, MRSA infection. If the excess length of stay occurred predominately before the manifestation of MRSA infection, this would likely represent the time at risk and therefore would be called a risk factor; if the excess length of stay occurred after MRSA infection, it would represent an associated outcome.

There are several important reasons for conducting case-control studies of antibiotic-resistant organisms. One

Dr. Perencevich is from the VA Maryland Healthcare System, Baltimore, Maryland. Dr. Hartley is from the Department of Epidemiology and Preventive Medicine, University of Maryland School of Medicine, Baltimore, Maryland.

Address reprint requests to Eli Perencevich, MD, MS, VA Maryland Health Care System, 100 N. Greene St, Lower Level, Baltimore, MD 21201. eperence@epi.umaryland.edu

Dr. Perencevich was supported by a VA Health Services Research and Development Service (HSR\&D) Research Career Development Award (RCD02026-1). Dr. Hartley was supported by NIH award K25AIO58956-02. 
reason for doing so is to identify a potentially modifiable risk factor that could be targeted for intervention. Another reason would be to identify, and then eliminate, a common exposure or source, thereby terminating the outbreak. In this study, however, the risk factors identified neither were easily modifiable nor identified a common source for the outbreak. So what did we learn? For one thing, all of the variables appeared to be highly correlated. It is probably reasonable to assume that neonates with a younger gestational age at birth would also have a lower birth weight, be more likely to require intubation and gavage feeding, and have a higher likelihood of birth secondary to a multiple gestation pregnancy. In fact, these factors have been associated either directly or indirectly with excess length of stay. ${ }^{68}$ Therefore, because several of these factors have biological plausibility in the causal pathway from uncolonized to colonized to infected (through their association with excess length of stay and severity of illness), some may prove effective candidates for inclusion in a prediction rule that would identify high-risk patients. ${ }^{9}$ Thus, an attractive next step in this population would be creation and validation of a prediction rule to detect patients at high risk for MRSA colonization for targeted active surveillance. ${ }^{10}$ If a high-risk population, defined as having certain risk factors for colonization, can be identified, this would lead to cost-effective active surveillance programs. Thus, colonization surveillance would not cease due to cost concerns after an outbreak has been controlled. In this outbreak, the authors reported eliminating active surveillance culturing 2 months after the outbreak was contained. Because our aim is to prevent the next outbreak, and not just halt one already in progress, cost-effective strategies must be identified.

Keene et al. describe the epidemiology of $S$. aureus (both MRSA and methicillin-sensitive $S$. aureus [MSSA]) colonization and infection in a cohort of patients in a medical ICU with an active surveillance program. ${ }^{5}$ Consistent with previous reports, colonized patients were far more likely to develop subsequent infection in the 30-day postdischarge period compared with uncolonized patients, and these infections were overwhelmingly identical to the initial colonizing strain. ${ }^{11-13}$ Importantly, $89 \%$ of the colonized patients were detected on admission, with the remaining detected during their ICU stay, presumably after acquisition in the ICU. These findings, along with their report of a rapid polymerase chain reaction (PCR) test with moderate sensitivity and high specificity, led the authors to suggest that colonized medical ICU patients may be optimal targets for a rapid detection and decolonization program. Unfortunately, their data provide evidence that a randomized trial testing such a program will be difficult and costly and may not achieve the desired outcome. Counterintuitively, colonized patients had lower ICU mortality (although not statistically significantly lower) than did non-colonized patients. In addition, those who developed infections did not have statistically significantly higher ICU mortality compared with those who did not develop infections. These findings are consistent with those from a large cohort study by Wertheim et al. that reported $S$. aureus bacteremia-related mortality was statistically significantly higher in non-carriers (32\%) than in patients found to be colonized on hospital admission (8\%). ${ }^{13}$ Therefore, an optimal strategy may be to prevent $S$. aureus colonization and infection in those not found to be already colonized on admission.

In contrast to the clinical epidemiology studies described in the articles by Khoury et al. and Keene et al., two other articles in this issue of Infection Control and Hospital Epidemiology describe mathematical modeling studies. ${ }^{2,3}$ Various models have appeared in the literature addressing different aspects of the antibiotic-resistance problem, including infection control and the benefits of various surveillance strategies. ${ }^{14-17}$ The articles by Forrester and Pettitt and Raboud et al. make valuable contributions to this literature.

Forrester and Pettitt consider statistical methods for estimating MRSA colonization transmission rates in ICU patients using interval-censored screening data. Their model partitioned patients into four states: (1) not colonized by MRSA, (2) colonized with MRSA but not isolated, (3) detected as colonized and isolated from other patients, or (4) removed or discharged from the ICU. Transitions between states were modeled as stochastic, or "random," events. Three competing sources of MRSA were included in the model: "background contamination"; non-isolated, colonized patients; and isolated, colonized patients. Background contamination was defined broadly to include nosocomial transmission arising from outside the ICU, MRSA carriers in the ICU who go undetected, colonized HCWs, and equipment and HCWs transiently contaminated within the hospital but outside the ICU. Importantly, it appears that the attributable impact on MRSA transmission of undetected and therefore non-isolated patients was analyzed separately and also included in the analysis of background contamination.

Results from the Forrester and Pettitt model suggest that, at least within the specific ICU considered, the transmission rate from background sources to susceptible patients was approximately double the rate of transmission per non-isolated patient and six times the rate of transmission per isolated patient. Thus, the model provided evidence to suggest that (1) background contamination is an important-and in this ICU, the dominant-source of incident colonization and (2) transmission from non-isolated MRSA patients via $\mathrm{HCWs}$ is greater than that from isolated MRSA patients. This last finding is consistent with the clinical belief that isolation of MRSA-positive patients is an effective control measure.

Raboud et al. describe a modeling study conceived to assess the impact of potential intervention strategies within a non-ICU setting, including adjusting the characteristics of screening tests, modifying nursing workload, varying handwashing rates, and the dependence of handwashing on the risk level of patients. Following an earlier model of Austin and Anderson, Raboud et al. allowed patients to exist in MRSA-colonized or MRSA-uncolonized states. ${ }^{18}$ In this model, "colonization" referred to either colonization or infection. Similarly, HCWs were assigned to either uncolo- 
nized or transiently colonized states; transitions between states were stochastic events.

The model suggests two approaches to reducing MRSA transmission-improving basic infection control measures and active screening in combination with control precautions-should be viewed as complementary. It predicts that establishing a culture-based admission screening and control program with an $80 \%$ compliance in performing admission surveillance cultures would lead to a $35 \%$ decrease in MRSA acquisition. Relative to this new baseline, introducing PCR-based rapid screening would lead to an additional $37 \%$ decrease, given that patients are isolated only when test results return positive (an "innocent until proven guilty" strategy). Increasing handwashing compliance by a small margin (10\%) projected a $50 \%$ decrease in MRSA acquisition. If the PCR test and increasing handwashing compliance by $10 \%$ were implemented simultaneously, the model predicts a decrease in nosocomial acquisition of more than $70 \%$. Consistent with previous conclusions drawn from Forrester and Pettitt, nurse-to-patient ratios were found to have little impact on MRSA transmission.

Besides the quantitative results found in these studies, how do the models inform our broader thinking about MRSA control? As with any modeling study, interpretation of the results depends on the assumptions with which the model is built. Raboud et al. assumed the dominant mode of MRSA transmission to be through hand contamination of HCWs. Transmission through direct patient-to-patient contact and environmental contacts was not included because, "Although these are possible routes of transmission, they are likely to be less important than hand contamination, and isolation precautions should also be effective in decreasing transmission by these routes." This appears to be in opposition to the finding of Forrester and Pettitt that background contamination was the dominant cause of incident colonization. However, several additional factors besides environmental contamination were included in Forrester and Pettitt's definition of background contamination, so the exact impact of environmental contamination remains unclear as far as the models are concerned. This highlights two of the key uses of models: to inform our thinking about potential interventions (Should we collect environmental swabs from commonly touched surfaces?) and data collection (What data do we need to collect to understand MRSA transmission from a mechanistic viewpoint?). Interestingly, Khoury et al. described environmental surface disinfection but did not collect surface samples to culture before or after cleaning.

To what degree are results drawn from a mathematical model generalizable? Raboud et al., for example, found that improving handwashing compliance by a small margin was likely to be an effective means of further reducing transmission of MRSA in their facility. Given this possibility, the next question to ask is: How sensitive is this finding to changes in individual parameters? Raboud et al. in fact provide some sensitivity analysis illustrating how model results vary according to a range of parameter values. However, the central parameters of the model, the relative probabilities for transmission during visits with and without handwashing and isolation, were not included in the sensitivity analysis. Moreover, these parameters were arrived at through expert opinion and were not empirically derived; as such, they may well be less supported by data published in the literature. Because these values may change from facility to facility as well as vary over time, it is both uncertain how to interpret the fundamental results of the model and difficult to assess what degree model results may be generalized to other institutions.

These four studies in this issue of Infection Control and Hospital Epidemiology provide a compelling illustration of how models and traditional epidemiologic studies complement one another. Traditional epidemiologic studies (such as cohort and case-control studies) are critically concerned with causal inference-the gathering of evidence on whether specific factors are indicative of a causal link to disease ${ }^{19}$ Mathematical models, on the other hand, assume causality (eg, improving handwashing compliance does reduce MRSA transmission) and predict the consequences of the assumption, allowing careful comparison with observation. ${ }^{20}$ Thus, modeling provides methods for interpreting observational data and testing hypotheses, such as the ability of a specific infection control intervention to halt a specific outbreak. Traditional epidemiologic studies must aim to maximize internal validity and minimize the role of chance in creating spurious results. ${ }^{21}$ Models, by providing a virtual workbench, can aid in epidemiologic study design because data can be collected on risk factors determined to be important in a mathematical model. Unfortunately, there is no perfect epidemiologic study, whether observational, experimental, or mathematical model simulation. In the case of infection control, despite common limitations, research studies provide opportunities to examine the effects of various components of control measures. By coordinating epidemiologic investigations, more of the "right" data can be collected with traditional cohort and case-control study methods and then analyzed using mathematical models, producing compelling results for policy decisions regarding hospital infection control.

Given the continued emergence of MRSA and other antibiotic-resistant bacteria in the hospital environment, infection control researchers must use the full armamentarium of study design methodologies at their disposal, including mathematical models and quasi-experimental designs. Randomized trials cannot be expected to answer all possible questions, given current cost and time constraints. Modeling studies provide a powerful methodology for designing future studies as well as interpreting data from them. We must constantly search for new study methodologies and control measures to evaluate and implement. The bacteria will not rest and neither will we.

\section{REFERENCES}

1. Wise RI, Ossman EA, Littlefield DR. Personal reflections on nosocomial staphylococcal infections and the development of hospital surveillance. Rev Infect Dis 1989;11:1005-1019.

2. Forrester M, Pettitt AN. Use of stochastic epidemic modeling to quantify transmission rates of colonization with methicillin-resistant Staphylococcus aureus in an intensive care unit. Infect Control Hosp 
Epidemiol 2005;26:598-606.

3. Raboud J, Saskin R, Simor A, et al. Modeling transmission of methicillin-resistant Staphylococcus aureus among patients admitted to a hospital. Infect Control Hosp Epidemiol 2005;26:607-615.

4. Khoury J, Jones M, Grim A, Dunne WM Jr, Fraser V. Eradication of methicillin-resistant Staphylococcus aureus from a neonatal intensive care unit by active surveillance and aggressive infection control measures. Infect Control Hosp Epidemiol 2005;26:616-621.

5. Keene A, Vavagiakis P, Lee M-H, et al. Staphylococcus aureus colonization and the risk of infection in critically ill patients. Infect Control Hosp Epidemiol 2005;26:622-628.

6. Elliott JP, Istwan NB, Collins A, Rhea D, Stanziano G. Indicated and non-indicated preterm delivery in twin gestations: impact on neonatal outcome and cost. J Perinatol 2005;25:4-7.

7. Apisarnthanarak A, Holzmann-Pazgal G, Hamvas A, Olsen MA, Fraser VJ. Ventilator-associated pneumonia in extremely preterm neonates in a neonatal intensive care unit: characteristics, risk factors, and outcomes. Pediatrics 2003;112:1283-1289.

8. Gilbert WM, Nesbitt TS, Danielsen B. The cost of prematurity: quantification by gestational age and birth weight. Obstet Gynecol 2003; 102:488-492.

9. McGregor JC, Kim PW, Perencevich EN, et al. Utility of the Chronic Disease Score and Charlson Comorbidity Index as comorbidity measures for use in epidemiologic studies of antibiotic-resistant organisms. Am J Epidemiol 2005;161:483-493.

10. Furuno JP, Harris AD, Wright MO, et al. Prediction rules to identify patients with methicillin-resistant Staphylococcus aureus and vancomycin-resistant enterococci upon hospital admission. $A m \mathrm{~J} \mathrm{In-}$ fect Control 2004;32:436-440.

11. Kluytmans J, van Belkum A, Verbrugh H. Nasal carriage of Staphylococcus aureus: epidemiology, underlying mechanisms, and associated risks. Clin Microbiol Rev 1997;10:505-520.
12. von Eiff C, Becker K, Machka K, Stammer H, Peters G. Nasal carriage as a source of Staphylococcus aureus bacteremia. $N$ Engl J Med 2001;344:11-16.

13. Wertheim HF, Vos MC, Ott A, et al. Risk and outcome of nosocomial Staphylococcus aureus bacteraemia in nasal carriers versus non-carriers. Lancet 2004;364:703-705.

14. Perencevich EN, Fisman DN, Lipsitch $M$, Harris AD, Morris JG $\mathrm{Jr}$, Smith DL. Projected benefits of active surveillance for vancomycin-resistant enterococci in intensive care units. Clin Infect Dis 2004;38:1108-1115.

15. Pelupessy I, Bonten MJ, Diekmann O. How to assess the relative importance of different colonization routes of pathogens within hospital settings. Proc Natl Acad Sci U S A 2002;99:5601-5605.

16. Bonten MJ, Austin DJ, Lipsitch M. Understanding the spread of antibiotic resistant pathogens in hospitals: mathematical models as tools for control. Clin Infect Dis 2001;33:1739-1746.

17. Austin DJ, Bonten MJ, Weinstein RA, Slaughter S, Anderson RM. Vancomycin-resistant enterococci in intensive-care hospital settings: transmission dynamics, persistence, and the impact of infection control programs. Proc Natl Acad Sci U S A 1999;96:69086913.

18. Austin DJ, Anderson RM. Studies of antibiotic resistance within the patient, hospitals and the community using simple mathematical models. Philos Trans R Soc Lond B Biol Sci 1999;354:721-738.

19. Rothman KJ, Sander G. Modern Epidemiology, ed. 2. Philadelphia: Lippincott-Raven; 1998.

20. Fine PEM. Applications of mathematical models to the epidemiology of influenza: a critique. In: Selby P, ed. Influenza Models: Prospects for Development and Use. Lancaster: MTP Press; 1982:15-85.

21. Shadish WR, Cook TD, Campbell DT. Experimental and Quasi-Experimental Designs for Generalized Causal Inference. Boston: Houghton Mifflin; 2002. 